\title{
Thermal Characterization of Carbon Nanotube Fiber by Time-Domain
}

\section{Differential Raman}

\author{
Changzheng $\mathrm{Li}^{1}$, Shen $\mathrm{Xu}^{2}$, Yanan Yue ${ }^{* 1}$, Bing Yang ${ }^{1}$, Xinwei Wang ${ }^{2}$ \\ ${ }^{1}$ School of Power and Mechanical Engineering, Wuhan University, Wuhan, Hubei 430072, China \\ ${ }^{2}$ Department of Mechanical Engineering, Iowa State University, Ames, IA, 50010, USA
}

\begin{abstract}
:
Most conventional Raman thermometry for thermal properties measurement is on steady-state basis, which utilizes either Joule heating effect or two lasers configurations coupled with increased complexity of system or measurement uncertainty. In this work, a new comprehensive
\end{abstract} approach including both transient and steady-state Raman method is proposed for thermal properties measurement of micro/nanowires. The transient method employs a modulated (pulsed) laser for transient heating and Raman excitation, and is termed time-domain differential Raman. The average elevated temperature during the transient heating period is probed simultaneously based on Raman thermometry. Thermal diffusivity can be readily determined by fitting normalized temperature rise against heating time with a transient heat conduction model. On the other hand, thermal conductivity can be obtained in the steady-state measurement by adjusting modulation settings. To verify this method, a carbon nanotube (CNT) fiber is measured with the thermal diffusivity of $1.74_{-0.20}^{+0.20} \times 10^{-5} \mathrm{~m}^{2} / \mathrm{s}$ and the thermal conductivity of $34.3_{-0.4}^{+0.4} \mathrm{~W} / \mathrm{m} \cdot \mathrm{K}$. The relatively low thermal transport values stem from numerous CNT-glue matrix and CNT-CNT thermal contact resistances. Compared with the conventional steady-state Raman method, the transient method requires no detailed laser absorption value and no temperature coefficient

*Corresponding author: E-mail: yyue@whu.edu.cn (Yanan Yue) 
calibration. It can be easily applied to study transient thermal transport in materials. 


\section{Introduction}

In recent years, Raman spectroscopy has been widely employed for temperature probing/mapping and thermal properties measurement [1-4]. Raman scattering is an inelastic scattering generated in laser-material interaction, and is temperature dependent in terms of peak intensity, peak shift, and peak width (full width at half maximum, FWHM) [5]. Therefore, Raman signals arising from temperature variation can be used to measure thermal properties of materials, such as in the studies of carbon nanotubes (CNTs) [6, 7], graphene [8-10], silicon [11] and other nano-materials $[12,13]$. For example, Zhang's group developed a non-contact T-type Raman spectroscopy method for the simultaneous measurement of micro/nano fibers' laser absorption and thermal conductivity [14]. Beechem et al. used micro-Raman spectroscopy to simultaneously map the complete temperature and biaxial stress distributions of a functioning polysilicon microheater [15]. Recently, they reported a comprehensive investigation of error and uncertainty in Raman thermal conductivity measurements based on numerical simulation [16]. In another work, Yue et $a l$. investigated the thermal response of a Si substrate under tip-induced near-field laser heating using Raman spectroscopy, and for the first time achieve the resolution down to sub-10 $\mathrm{nm}$ [17].

Most optical thermometry techniques for thermal diffusivity determination are transient methods, such as laser flash method and time-domain thermoreflectance (TDTR, or pump- probe) method etc [18-22]. The thermal diffusivity, which is based on transient heat transfer model, is usually obtained in these optical thermal characterizations. Different from abovementioned optical thermometry, Raman thermometry works as a steady-state measurement tool since the continuous 
probe laser is employed and a relative long integration is required for a sound Raman signal. Steady-state heat transfer models are introduced for thermal transport description and precise measurement of heat flux is needed to calculate thermal conductivity of the material. In Yue et al.'s works [2, 23], a steady-state electro-Raman-thermal (SERT) technique was developed to characterize thermal transport in micro/nanoscale materials. In this method, the middle-point temperature of a microwire was recorded using Raman spectroscopy when the sample was heated by electric current. Thermal conductivity was derived from the relationship between the middle-point temperature and the Joule heating energy. However, the electrical/thermal contact resistance at sample-electrode conjunctions, and additional heating effect from the probing laser would contribute to unexpected measurement errors. Moreover, the electric circuit and electrodes are difficult to design for micro/nanoscale materials. Besides electrical heating, laser heating was employed to induce temperature difference for steady-state Raman spectroscopy measurements [3, 24-26]. This method eliminates the fabrication difficulties in electrical heating method and the highly focused laser spot shows great advantages in thermal probing at extremely small scales. But we need the precise calculation of laser absorption for calculating thermal conductivity. This requires the knowledge of the specific laser absorbance at certain wavelengths for the measured materials, which is usually unknown for novel materials [7].

Therefore, developing an optical method which is capable of measuring both thermal conductivity (steady-state thermal characterization) and thermal diffusivity (transient thermal characterization) is necessary and significant. Conventional Raman thermometry are based on 
continuous laser for heating and temperature probing, which gives us an idea that if using pulsed laser instead for Raman signal excitation, transient Raman thermometry could be possible [27, 28]. Furthermore, if the excitation laser is adjustable from continuity to pulse, both steady-state measurement and transient characterization based on Raman thermometry can be achieved. Recently, it was reported that a time-domain differential Raman (TD-Raman) thermometry was developed by modulating the excitation laser and probing transient Raman scattering during the pulsed heating circle [29]. In this work, the thermal diffusivity of a silicon cantilever was measured to validate the measurement capacity. However, most industrial materials do not have such a good crystalline structure as single crystalline silicon. The lower Raman excitation efficiency due to their less crystalline structure would increase the difficulty of the TD-Raman measurement. For example, the carbon nanotubes fiber (CNT fiber), which is a scale-up material from single CNTs, presents exceptional macroscopic properties. It has wide applications and is of great interest to explore its thermal properties [30-33]. In this work, we use TD-Raman method to measure the thermal diffusivity of CNT fiber, and combine the steady-state Raman method to study its thermal properties comprehensively. Most importantly, we expand the application of the recently developed transient Raman method [29], and achieve both transient measurement and steady-state thermal characterization based on Raman thermometry at the same experimental configuration.

\section{Experimental principle and details}

\subsection{Experimental principle}


Fig. 1 illustrates the schematic setup of this Raman thermal characterization. A sample is suspended between two heat sinks in a vacuum chamber (to minimize heat convection effect). The heat sinks have large heat capacity and high thermal conductivity, ensuring the ends of the sample stay at room temperature. This approach contains transient and steady-state measurements which share the same configuration. For transient measurement, a continuous wave laser is modulated with an electric-optical modulator to generate square-wave pulse laser with variable laser-on durations. It is then focused on the middle point of the sample. The pulse laser consists of an excitation period $\left(t_{\mathrm{e}}\right)$ followed by a thermal relaxation period $\left(t_{\mathrm{r}}\right)$. When the pulse laser is on in the excitation period, the sample is heated and its temperature rises. During this period, the spectrometer's shutter opens and Raman scattering is accumulatively collected. The collected Raman signal is an integration of instant temperature information over the heating period. The thermal relaxation period is long enough and allows the sample to cool down to the ambient temperature before the next excitation cycle starts. Sufficient "heating and cooling" cycles are repeated (by adjusting integration time) to obtain a sound Raman spectrum signal for temperature measurement. 


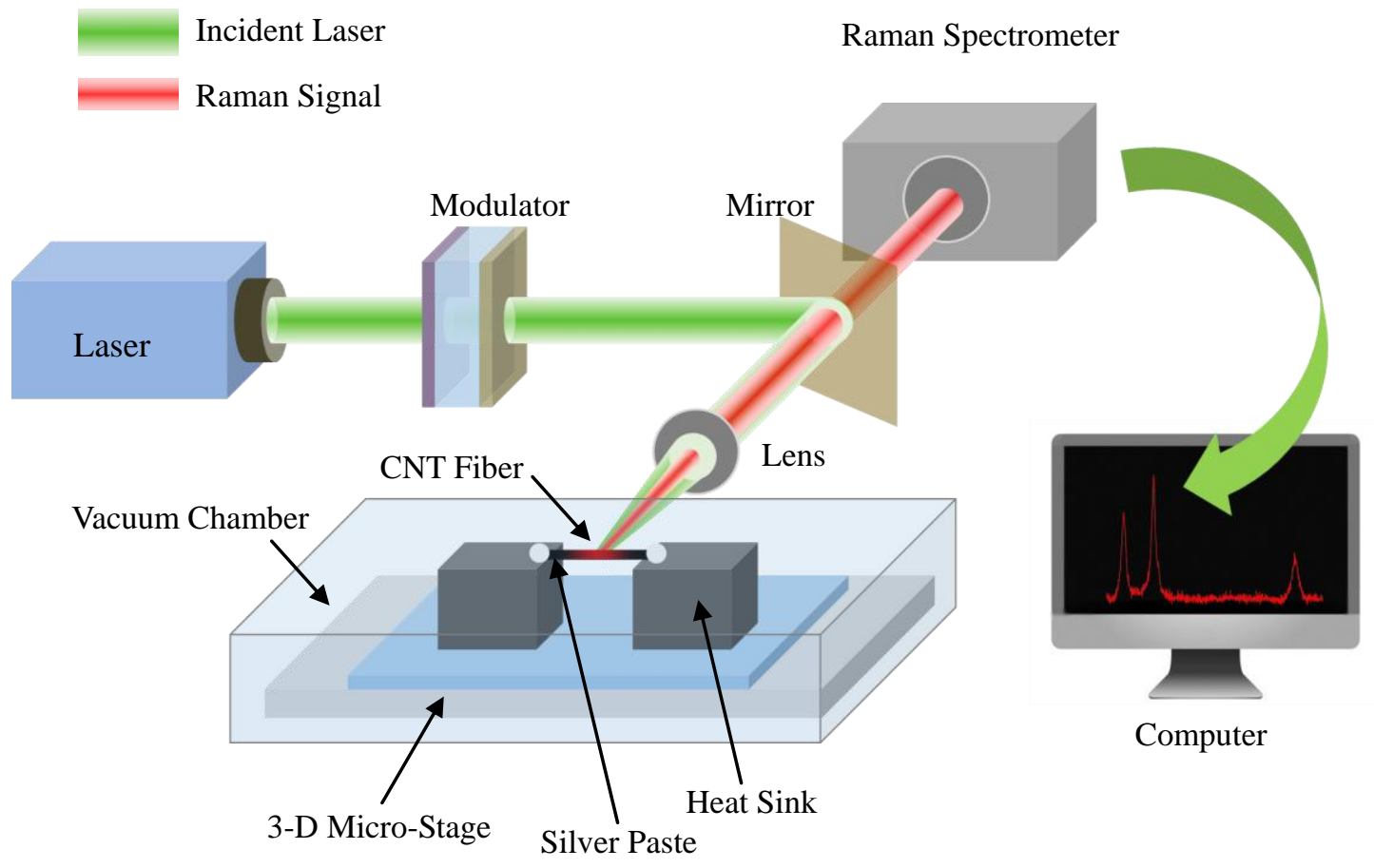

Fig. 1 The schematic of the TD-Raman method for thermal characterization of CNT fiber. A continuous wave laser is modulated with an electric-optical modulator to output a square pulse laser and focused on the middle point of the sample for both heating and Raman excitation. Raman signal is recorded by a Raman spectrometer for temperature measurement.

The physical model of this transient Raman technique is presented in Fig. 2a. In the experiment, the laser spot can cover the whole sample in the diameter direction. As the length of the sample is much longer than the diameter, a one-dimensional (1-D) model is established for interpreting the heat transport along the sample with the governing equation as

$$
r c_{p} \frac{\partial q}{\partial t}=k \frac{\partial^{2} q}{\partial x^{2}}+q_{0},
$$

where $\rho$ and $c_{p}$ is the density and the specific heat of the sample, respectively. $\theta$ is the 
temperature rise, $k$ is thermal conductivity, $x$ is the distance away from the heat sink, $q_{0}$ is the heat generation per unit volume induced by the laser heating. Considering the symmetry, we take half of the sample to discuss thermal conduction in the sample, as shown in Fig. 2a. Combined with the boundary conditions, the spatially averaged temperature rise in the heated region can be expressed as [29]

$$
\bar{q}(t)=\frac{2 q_{0} L^{3}}{k\left(x_{2}-x_{1}\right)} \underset{m=1}{\stackrel{\ddagger}{a}} \frac{1}{m^{4} p^{4}}\left[1-\exp \left(-\frac{m^{2} p^{2} a t}{L^{2}}\right)\right]\left(\cos \frac{m p}{L} x_{1}-\cos \frac{m p}{L} x_{2}\right)^{2} .
$$

where $\alpha$ is the thermal diffusivity of the sample, $L$ is the length of the sample. The coordinates of $x_{1}$ and $x_{2}$ are shown in Fig. 2a. Fig. 2b and 2c show three selected experimental Raman spectra of the CNT fiber sample under different excitation times $\left(t_{\mathrm{e}}\right)$ in one "heating and cooling" cycle. As excitation time is increased, the Raman peaks (D band and G band in Fig. 2c) shift to shorter wavenumber direction. This shift is attributed to the temperature rise at the heating point with $t_{\mathrm{e}}$ increasing. The Raman intensity also increases because of the longer excitation time and so as the scattering collection time. The average temperature rise in the heated region can be obtained from the measurement Raman signal. The thermal diffusivity of the sample can be fitted from the normalized temperature rise against heating time as

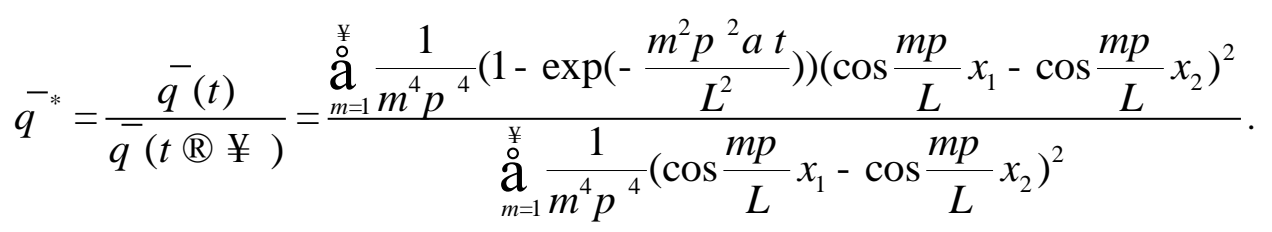

For the steady-state measurement, a continuous wave laser directly irradiates on the middle point of the sample and heat dissipates from center to the heat sinks. When the sample reaches the 
steady state, the average temperature within laser irradiation region can be expressed as [34]

$$
\bar{T}=\frac{Q L}{4 p k r_{s}^{2}}-\frac{Q r_{0}}{3 p k r_{s}^{2}}+T_{0}
$$

where $Q$ is absorbed laser energy $\left(Q=2 q_{0} \pi r_{\mathrm{s}}^{2} r_{0}\right), T_{0}$ is room temperature, $r_{\mathrm{s}}$ and $r_{0}$ are the radius of the sample and the laser spot, respectively. Thermal conductivity of the sample can be readily derived by measuring the average temperature of the heated region with Raman spectroscopy. This novel Raman method features noncontact, non-destructive and high spatial resolution in both transient and steady-state thermal properties measurements and the absorbed laser energy is not needed for measuring thermal diffusivity with TD-Raman method. 
(a)
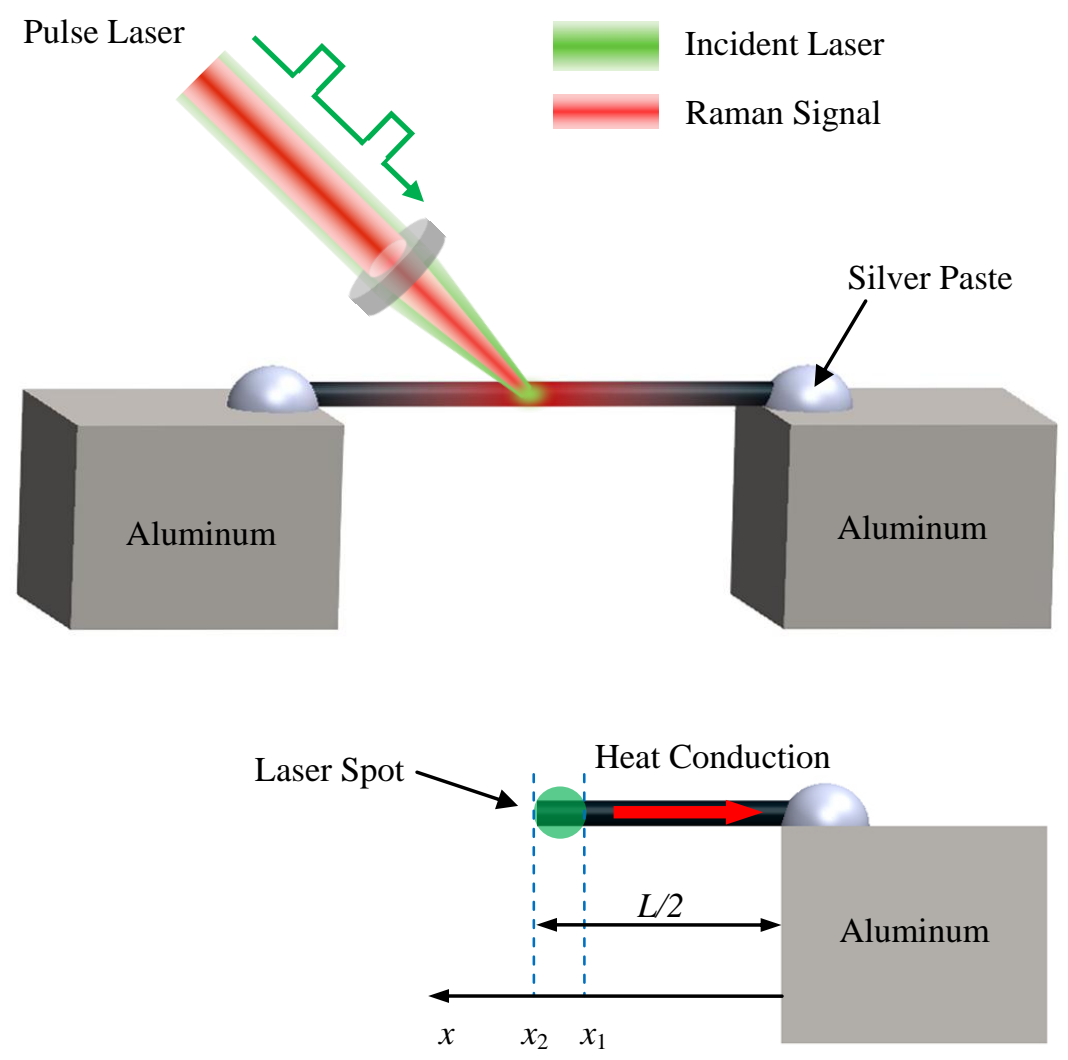

(b)
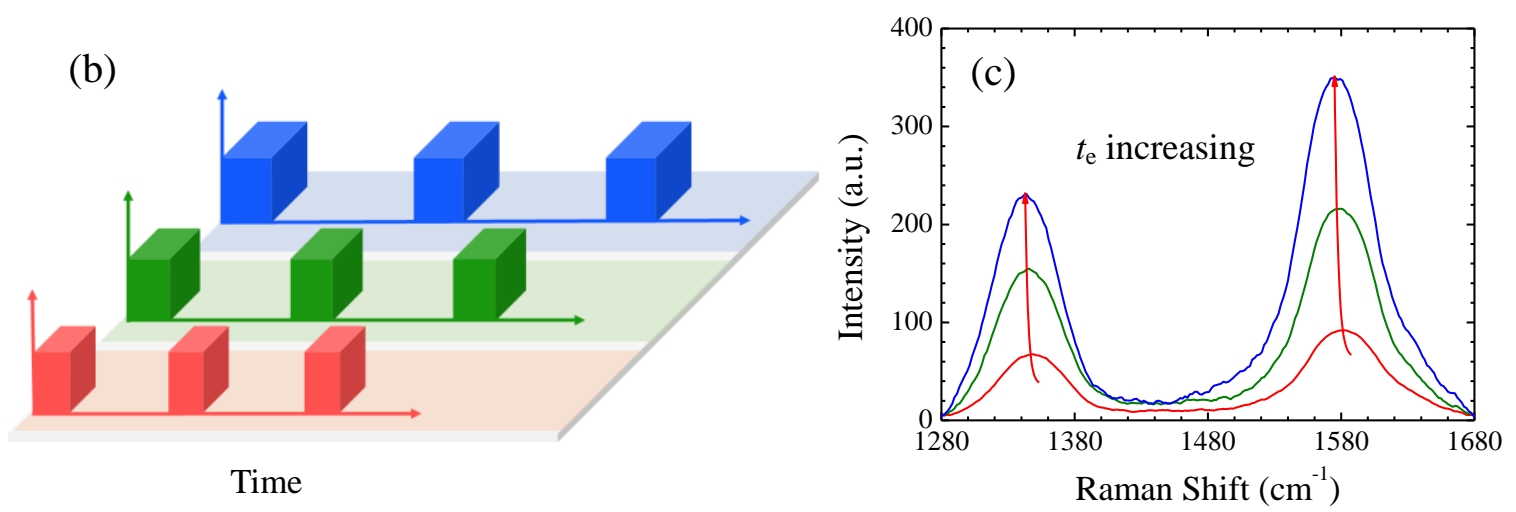

Fig. 2 (a) Physical model used for transient Raman measurement. The corresponding coordinates are marked in the physical model. (b) Three selected square pulse lasers of different excitation time $\left(t_{\mathrm{e}}\right)$ for both heating and Raman excitation measurement. (c) The corresponding Raman spectrum of the CNT fiber sample for one "heating and 
cooling" cycle. The Raman peaks shift to shorter wavelengths and the Raman intensity increase as the excitation time is increased.

\subsection{Experimental details}

The CNT fiber sample used in this work was purchased from Nanjing JCNANO Company prepared by a floating catalyst method. In the measurement, the CNT fiber is suspended between two aluminum heat sinks with both ends connected by silver paste. The conjunctions are carefully examined to make sure thermal contact resistance can be ignored. The sample is placed on a micro-stage in a vacuum chamber with the atmosphere pressure under $10^{-3}$ Torr to eliminate heat convection. Fig. 3a presents the sample placed on a micro-stage with a length of $2 \mathrm{~mm}$. Fig. 3b and $3 c$ are the scanning electron microscopy (SEM) images of the CNT fiber. Fig. 3b shows the surface morphology and Fig. $3 \mathrm{c}$ shows the inner structure at the break point under stretching. It is shown that the CNT fiber consists of thousands of individual CNTs which twist tightly. The diameter $\left(D=2 r_{\mathrm{s}}\right)$ of the CNT fiber is determined to be $36 \mu \mathrm{m}$ averaged from multiple measurements. The high ratio of length to diameter means that the 1-D model is applicable in this case according to previous theoretical analysis. 

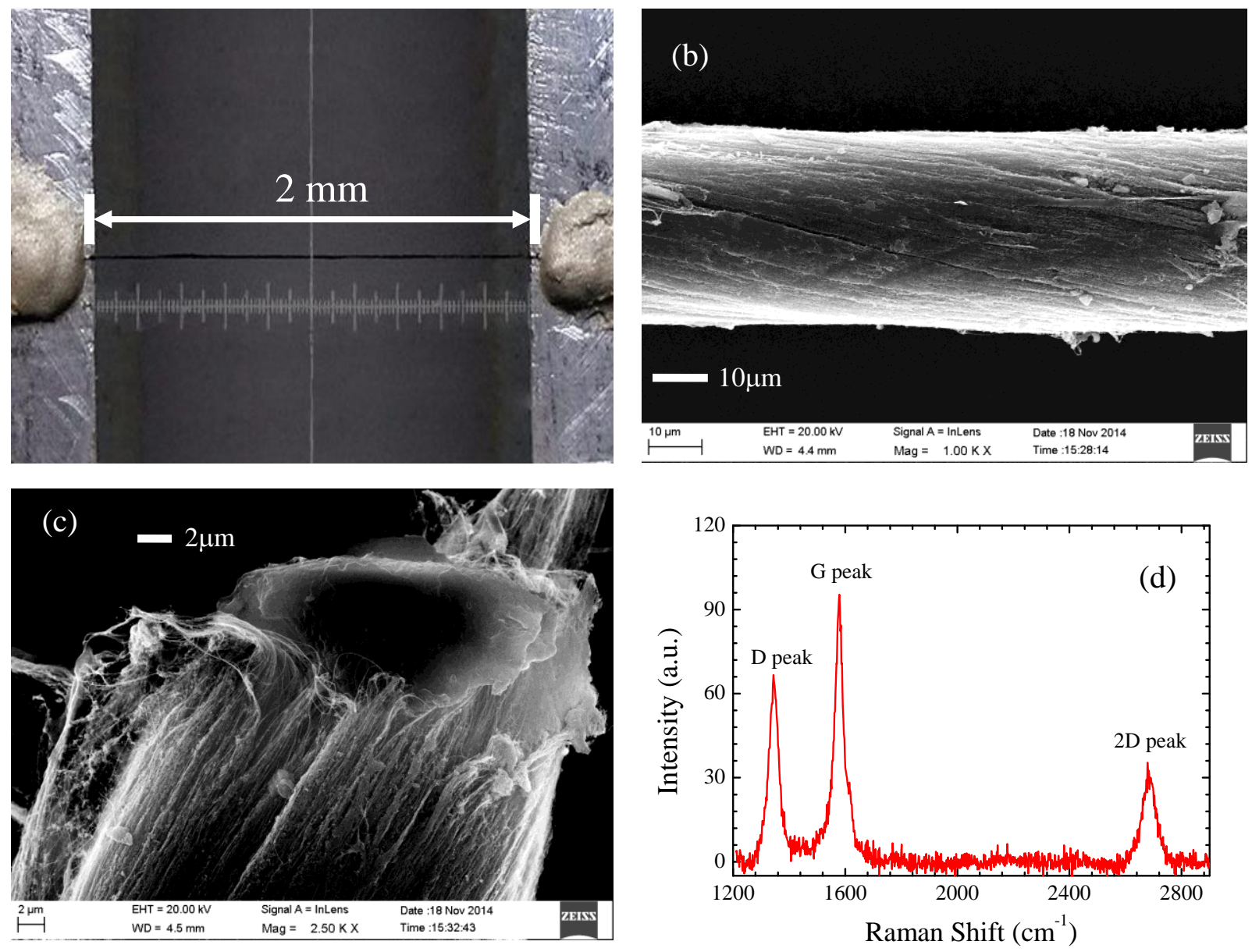

Fig. 3 (a) The sample image of CNT fiber with ends adhered between two aluminum heat sinks. The length of the measured sample is $2 \mathrm{~mm}$. (b-c) The SEM images of the CNT fiber. It shows the surface morphology and the inner structure at the break point respectively. (d) The Raman spectra of CNT fiber sample, strong Raman signal with three identified peaks about $\sim 1350 \mathrm{~cm}^{-1}, \sim 1580 \mathrm{~cm}^{-1}$ and $\sim 2700 \mathrm{~cm}^{-1}$. Intensity of $\mathrm{G}$ band and $2 \mathrm{D}$ band reveals that the composite CNT fiber is consisted of high volume MWCNTs. The strong intensity of D band means that CNTs have high defect level.

Fig. 3d presents a typical Raman spectrum of the CNT fiber sample: strong Raman signal with three identified peaks about $\sim 1350 \mathrm{~cm}^{-1}, \sim 1580 \mathrm{~cm}^{-1}$ and $\sim 2700 \mathrm{~cm}^{-1}$ can be identified explicitly, 
corresponding to the D band (disorder), $\mathrm{G}$ band (graphite) and 2D band (second-order harmonic). Intensity ratio of $\mathrm{G}$ band and 2D band reveals that the composite CNT fiber consists of multiwall CNTs with a high weight fraction [35-37]. The strong-disorder-induced D-band intensity means that this CNT fiber contains high intrinsic defects of CNTs. Considering the rugged surface of the CNT fiber (as shown in Fig. 3b), the thermal expansion and the focal levels of the optical path will affect the peak intensity. Also the peak width variation is sometime too week for temperature measurement $[27,29]$. In this work, Raman peak shift is used for temperature determination.

A calibration experiment is first conducted to determine temperature coefficient of the peak shift. Raman spectrum is recorded with the sample being heated from $14.4{ }^{\circ} \mathrm{C}$ to $123{ }^{\circ} \mathrm{C}$. Gaussian function is used to fit the Raman spectrum and get the peak position of the same CNT fiber sample. Previous studies have shown that Raman shift is in a linear relationship with temperature for an individual CNT within a small temperature range $[6,38]$. In our experiment, the CNT fiber comprises of single CNTs, thus the relationship between Raman peak position and the corresponding temperature can be regarded as linear within the temperature range of our experiment. Through linear-fitting, the temperature dependence coefficient for the CNT fiber is obtained.

In the transient measurement, the electric-optical modulator is turned on. The continuous laser is modulated to generate variable square-wave pulse laser and then focused on the middle of the sample. The laser spot is determined to be circular with a radius $\left(r_{0}\right)$ of $50 \mu \mathrm{m}$ using the general 
knife-edge method. Different from previous work [29], the duty ratio $\left(t_{\mathrm{e}} /\left(t_{\mathrm{e}}+t_{\mathrm{r}}\right)\right)$ of the square-wave pulse laser is set to be $5 \%$ for all measurements. The duration of the heating period in one cycle is adjusted from $5 \mathrm{~ms}$ to $340 \mathrm{~ms}$ by changing the modulation frequency, e.g. $10 \mathrm{~Hz}$ for $5 \mathrm{~ms}$ heating $\left(t_{\mathrm{e}}=\right.$ duty/frequency). The characteristic time of the heat transport along the sample can be estimated as $0.81 l^{2} / \alpha[29,39]$, where $l$ is half length of the sample $(l=L / 2)$. With the referenced thermal diffusivity of $1.51 \times 10^{-5} \mathrm{~m}^{2} / \mathrm{s}$ at $39{ }^{\circ} \mathrm{C}[40]$, the characteristic time of the CNT fiber is about $53.6 \mathrm{~ms}$. The shortest thermal relaxation time for the case of $5 \mathrm{~ms}$ heating experiments is $95 \mathrm{~ms}\left(t_{\mathrm{r}}=t_{\mathrm{e}} /\right.$ duty- $\left.t_{\mathrm{e}}\right)$. It ensures that the sample can be cool down to room temperature within every "cooling" cycle. Much longer relaxation time for the measurements of longer heating time ensures that there is no heat accumulated in every single cycle for all different heating experiments. For steady-state measurement, the continuous laser is focused on the middle point of the sample. The absorbed irradiating laser energy on the sample is $13.75 \mathrm{~mW}$. This small energy input can produce enough temperature rise in the sample for the thermal conductivity measurement but not damage the sample. The Raman signal integration time is set to be $300 \mathrm{~s}$ for all the measurements.

\section{Results and discussions}

\subsection{Thermal characterization results of CNT fiber}

Fig. $4 \mathrm{a}$ is the schematic illustration of the temperature rise when sample is irradiated by the square-wave pulse laser in the transient measurement. During the excitation period, the temperature increases quickly when the laser is on. The average temperature of this period is 
measured using $\mathrm{G}$ band peak position. Fig. 4b shows the G-band peak shift against the excitation time. It decreases quickly at the beginning of the laser heating time, meaning that the temperature increases rapidly before the heat transfers to the electrodes. As the heating time is increased, the shift variation slows down until being a constant for the sample reaches the steady state. Noticing the good linearity between the peak shift and temperature, the normalized temperature rise of the CNT fiber can be obtained from

$$
\bar{q}^{*}=\frac{w(t)-w_{0}}{w_{s}-w_{0}},
$$

where $\omega(t)$ is the peak shift at different heating time, $\omega_{0}$ and $\omega_{\mathrm{s}}$ are the peak shift of the sample measured at room temperature and steady state, respectively. Fig. $4 \mathrm{c}$ shows the relationship between the normalized temperature increase and the heating time. The thermal diffusivity of $1.74_{-0.20}^{+0.20} \cdot 10^{-5} \mathrm{~m}^{2} / \mathrm{s}$ is obtained by using the least square fitting method with Eq. (4) by transient Raman method. Using this measured thermal diffusivity to obtain the real characteristic time of $46.6 \mathrm{~ms}$ confirms that indeed there is no heat accumulated for all different heating experiments. 
(a)

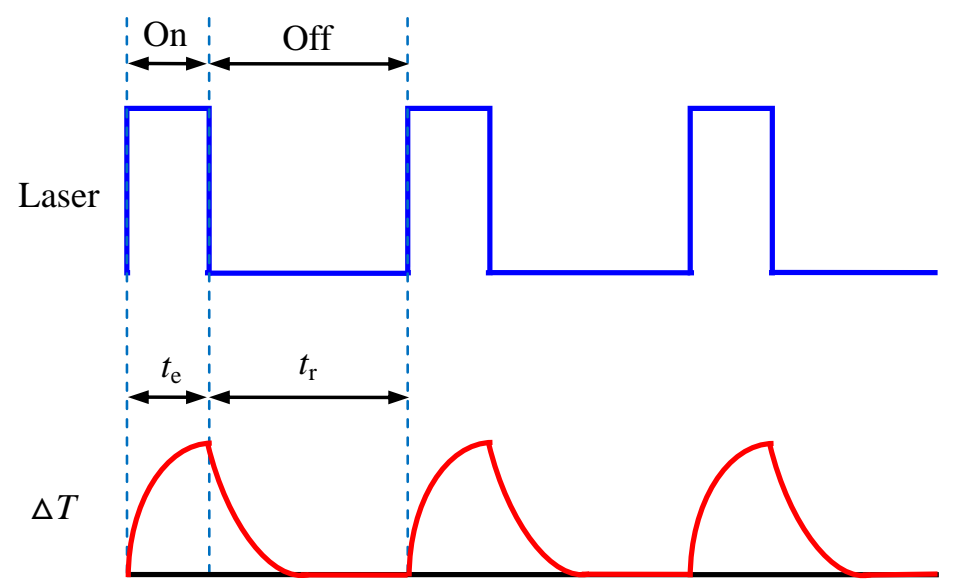

Time

(b)

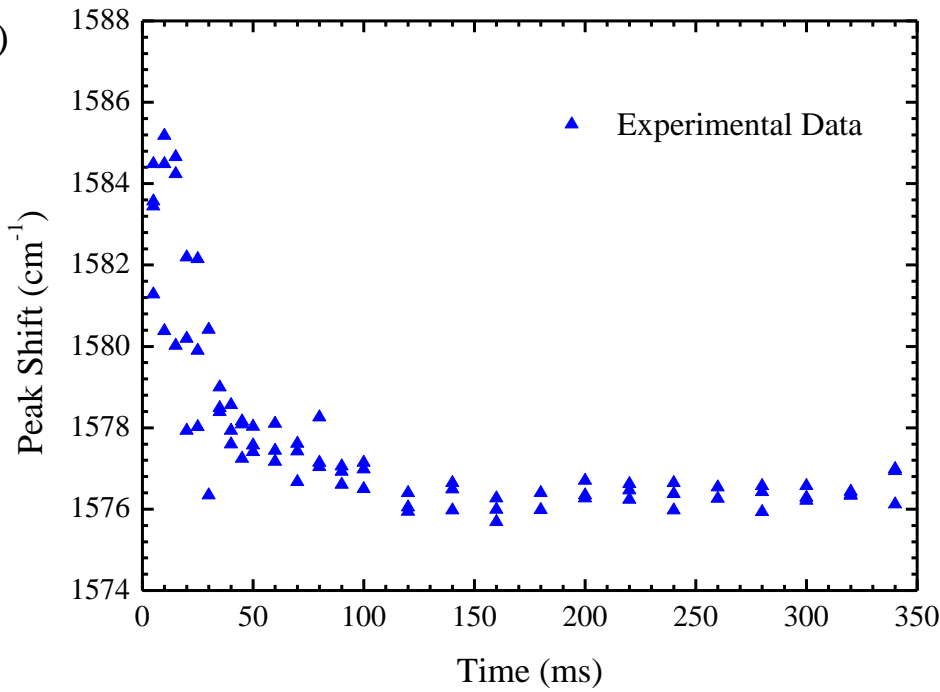

(c)

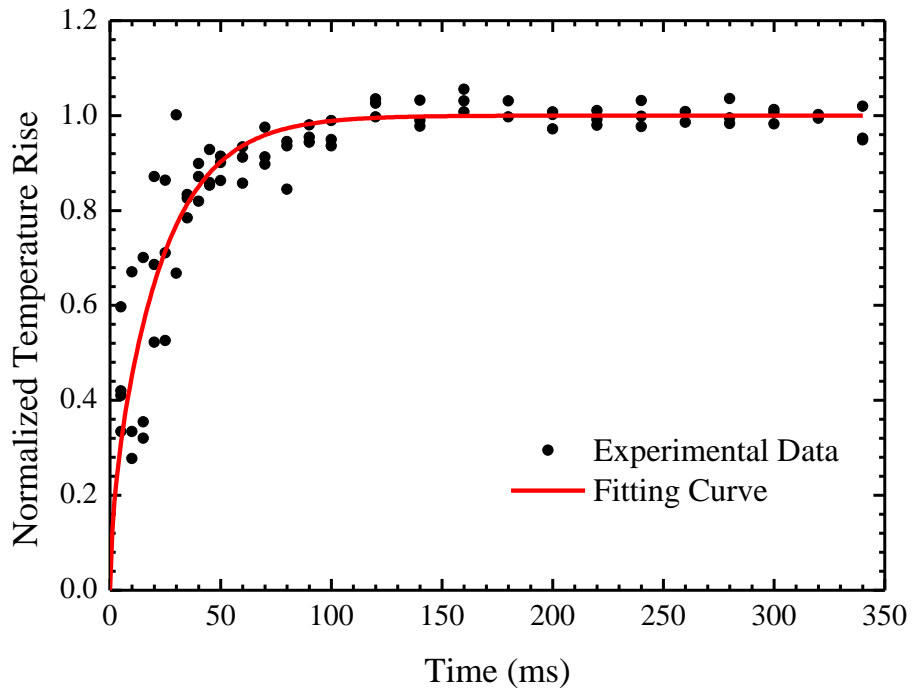

Fig. 4 (a) The schematic illustration of temperature rise when the sample is irradiated by the square pulse laser. The 
temperature of sample rises quickly when the laser is on and the sample is cooled down to the room temperature in the thermal relaxation period. (b) The peak shift to the steady sate against the heating time. (c) The normalized temperature rise and the fitting curve for measuring the thermal diffusivity of CNT fiber.

The TET (transient electrothermal) technique, which is a well-developed method and has been employed in many characterizations $[39,41,42]$ is used to verify the measurement results of the new method in this work. In TET technique, the sample is suspended between two electrodes and heated by a step current as shown in Fig. 5a. The thermal diffusivity can be fitted from the transient process of temperature rising using

$$
\bar{q}^{*}=\frac{96}{\pi^{4}} \underset{m=1}{\stackrel{\ddagger}{\circ}} \frac{1-\exp \left[(-2 m-1)^{2} \pi^{2} \alpha t / L^{2}\right]}{(2 m-1)^{4}} .
$$

More details for the principle about this technique can be found in Guo et al.'s work [39]. In the measurement, a step current of $22 \mathrm{~mA}$ is applied to the same CNT fiber. The temperature rise of the sample is extracted from the resistance change by measuring voltage with a data acquisition card (NI USB-6009). To reduce the measurement errors, three experiments were taken for averaging. Fig. 5b shows the experimental data and best fitting curve for the normalized temperature rise based on Eq. (6). Thermal diffusivity of the CNT fiber measured by TET method is determined to be $1.76_{-0.06}^{+0.06} 10^{-5} \mathrm{~m}^{2} / \mathrm{s}$, which is in good agreement with the value $\left(1.74_{-0.20}^{+0.20} 10^{-5} \mathrm{~m}^{2} / \mathrm{s}\right)$ measured by the transient Raman method. A slight difference may be caused by the different temperatures of the sample during measurements, which would affect the thermal property [41]. 
(a)

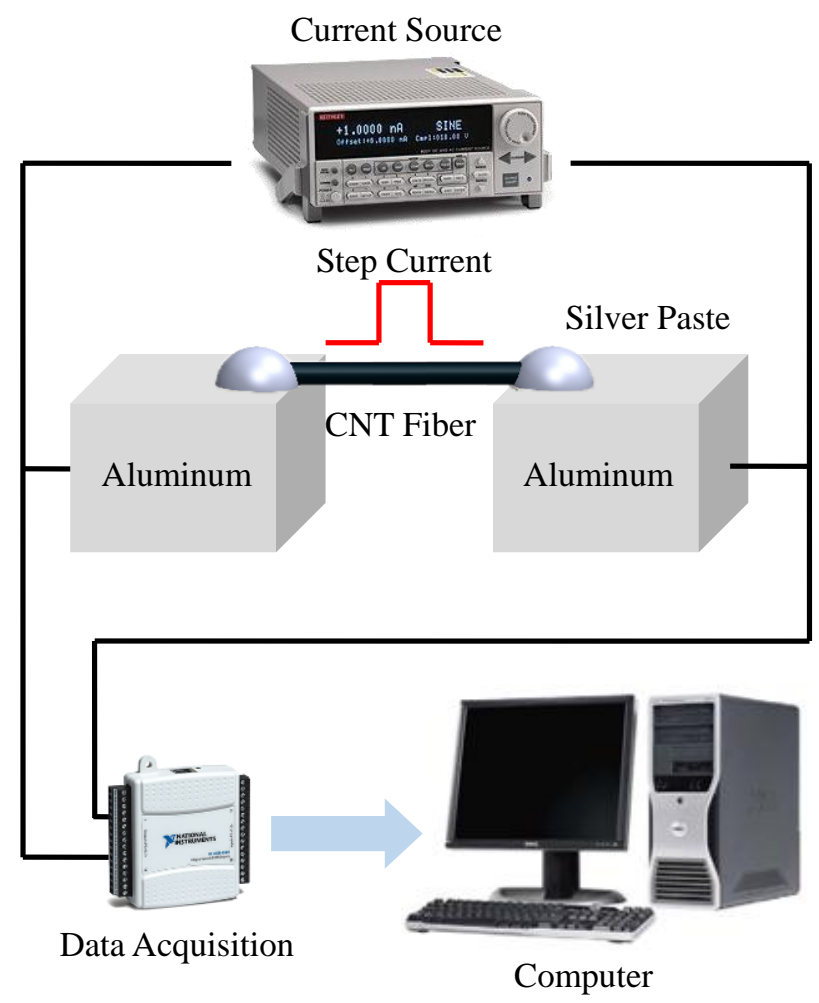

(b)

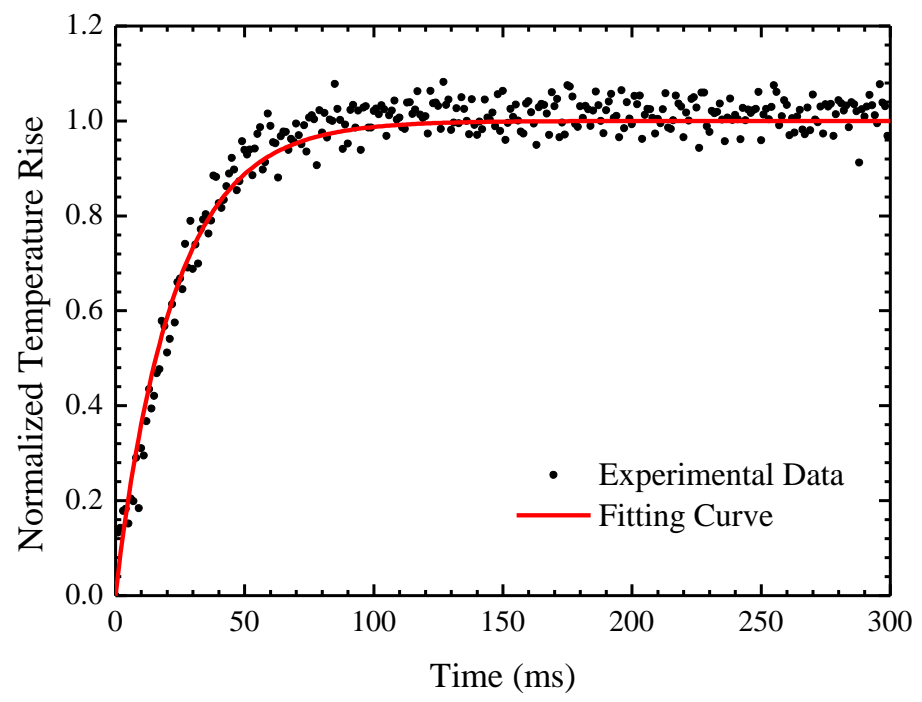

Fig. 5 (a) Schematic of the TET technique and experimental setup. (b) The experimental data and best fitting curve for the normalized temperature rise in TET method. The thermal diffusivity of the CNT fiber is determined as $1.74_{-0.20}^{+0.20} 10^{-5} \mathrm{~m}^{2} / \mathrm{s}$. 
Thermal conductivity of the CNT fiber can be obtained in the steady-state measurement. Fig. 6a shows the relationship between the Raman shift of G band and the corresponding temperature. The temperature dependence coefficient is fitted to be $-0.0486 \mathrm{~cm}^{-1} /{ }^{\circ} \mathrm{C}$. Fig. $6 \mathrm{~b}$ indicates the Raman spectrum of the sample and how double-peak fitting (red solid line) results in peak positions. Two Raman peaks of D band and G band can be obtained as $1346.15 \mathrm{~cm}^{-1}$ and 1578.34 $\mathrm{cm}^{-1}$. The average temperature on the irradiated middle point of the sample can be decided according to the calibration experiment of $\mathrm{G}$ band peak position. The determined thermal conductivity of the CNT fiber is $34.3_{-0.4}^{+0.4} \mathrm{~W} / \mathrm{m} \cdot \mathrm{K}$ according to Eq. (4). As thermal diffusivity is defined as $\alpha=k / \rho c_{p}$, combining with the thermal diffusivity measured by the transient method, an important parameter of $\rho c_{p}$ is determined to be $1965 \mathrm{~kJ} / \mathrm{m}^{3} \cdot \mathrm{K}$. This value, also termed volumetric heat capacity (VHC), is the amount of heat needed to raise the temperature of per unit volume by one degree Celsius, describing the ability of the CNT fiber to store internal energy while undergoing a given temperature change. In the previous work, Xie et al reported that the VHC $\left(\rho c_{p}\right)$ of multiwalled carbon nanotube arrays was about $1170 \mathrm{~kJ} / \mathrm{m}^{3} \cdot \mathrm{K}$ [43], which is in the same order with our value. 

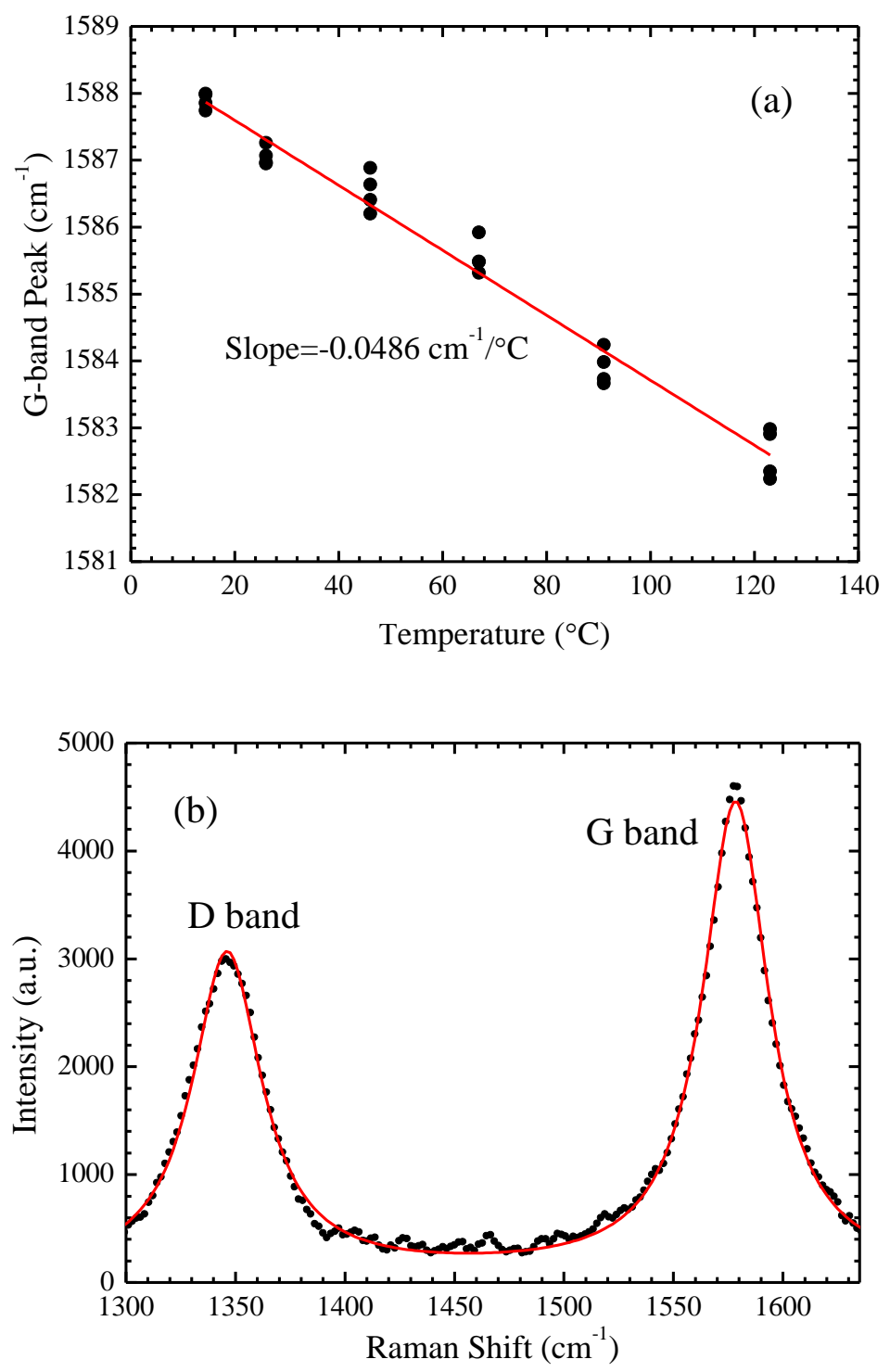

Fig. 6 (a) The linear fitting of $\mathrm{G}$ band peak positions and corresponding temperature. The slope of the fitting line (red solid line) is determined as $-0.0486 \mathrm{~cm}^{-1} /{ }^{\circ} \mathrm{C}$. (b) The Raman spectrum of the sample at steady-state measurement and the double-peak fitting curve (red solid line).

The very low thermal conductivity of CNT fiber in comparison with individual CNT arises from CNT-CNT thermal contacts resistance, which has been evidenced in our previously studies [40, 
42]. Mechanical stretching and temperature rise can decrease the contact resistance and thus increase the thermal conductivity. Since temperature is an important factor which could significantly affact thermal property of the material, it is important to know the temperature of sample during measurement. The steady state temperature rise $\Delta T$ can be calculated with the steady state heat conduction equation $q=k A \Delta T / l$. Taking the half of the sample for analysis, as shown in Fig. 2a, heat flux $q$ equals to half of the absorbed laser energy $(Q / 2), k$ is the thermal conductivity measured by steady-state method $\left(34.3_{-0.4}^{+0.4} \mathrm{~W} / \mathrm{m} \cdot \mathrm{K}\right), A$ is the cross-section area of the sample and $l$ is half length of the sample. The steady state temperature rise of the middle point of the sample is about $200{ }^{\circ} \mathrm{C}$. It is in good agreement with the thermal conductivity of the same CNT fiber sample measured by steady-state Joule-heating and Raman mapping in our previous work $\left(34 \mathrm{~W} / \mathrm{m} \cdot \mathrm{K}\right.$ in temperature of $\left.195{ }^{\circ} \mathrm{C}\right)$ [40], considering different experimental conditions.

\subsection{Uncertainty analysis of measurement results}

The uncertainty of measurement results mainly stems from two parts: heat loss from the sample surface and errors of measurement. Heat loss is caused by thermal radiation and convection. Due to very low pressure $\left(10^{-3} \mathrm{Torr}\right)$ in the vacuum chamber, the heat convection of the sample surface can be neglected. The thermal radiation induced heat loss from the sample surface in the transient method can be evaluated using the equation $16 \varepsilon \sigma T_{0}^{3} L^{2} /\left(\rho c_{p} D \pi^{2}\right)$ [44]. Where $\varepsilon$ is the effective emissivity of the sample (taking $\varepsilon=1$ for the maximum calculation), $\sigma$ is the Stefan-Boltzmann constant, $D$ is the diameter of the CNT fiber sample. The radiation effective thermal diffusivity is then determined to be $\sim 1.63 \times 10^{-7} \mathrm{~m}^{2} / \mathrm{s}$. This causes only about $1 \%$ deviation in the transient 
Raman measurement. In our measurement, aluminum-foil was used to cover the vacuum chamber to ensure that the measurement uncertainty from radiation heat loss can be ignored. Another measurement uncertainty mainly comes from the dislocation of middle point measurement. This will cause errors for $L, x_{1}$ and $x_{2}$ in Eq. (3). Assuming the laser heating point is $10 \%$ deviated from the middle point of the sample. Re-fitting with Eq. (3), we get a new thermal diffusivity as $1.74_{-0.20}^{+0.20} 10^{-5} \mathrm{~m}^{2} / \mathrm{s}$. The error is within $0.3 \%$. In fact, the micro-stage has a high lateral resolution of $1 \mu \mathrm{m}$, compared with the sample length of $2 \mathrm{~mm}$, the possible dislocation is far less than the assumed one. Therefore the small middle point deviation in measurement does not cause large errors.

In addition, this new Raman method does not use Joule heating thus no electrical setup is needed. In the transient measurement, a pulse laser is used for both heating and Raman excitation measurement. The absorbed energy and the slope in Raman signal calibration experiment is not necessary in the fitting process for thermal diffusivity measurement. It only requires the sample have good linear relationship between Raman shift and temperature which can be achieved by controlling the measurement within a small temperature range. Besides the well-developed steady state Raman-based methods' advantages, this new method can be used to measure the thermal diffusivity directly, and fasten measurement process. This method shows great superiority especially in micro/nano thermal probing. Since this technique is based on Raman scattering which is non-elastic scattering and the signal is very weak. Therefore, time-domain differential Raman method can but only applied on Raman-active materials. If to-be-measured material is not 
Raman-active, it is suggested to coat additional layer of Raman-active materials as the temperature indicator for implementation of this technique. And of course the measurement uncertainty introduced from the coating material needs to be well controlled.

In current work, the CNT fiber sample is used to examine this method's capability. It is worth to note that this new method is also suitable for measuring bulk material and interface materials. For bulk materials, we can use semi-infinite thermal model for describing the transient thermal transport. For interface measurement, e.g., the monolayer material interface, which requires high temperature sensitivity, ultra-fast laser can be used for transient Raman excitation and probing, which could be an excellent direction to make attempt.

\section{Conclusion}

In summary, a comprehensive approach combining steady-state and transient Raman thermometry is employed to measure the thermophysical properties of CNT fiber. The thermal diffusivity of $1.74_{-0.20}^{+0.20} 10^{-5} \mathrm{~m}^{2} / \mathrm{s}$ was determined by fitting the normalized temperature rise against heating time with transient measurement by adjusting modulation settings. Thermal

conductivity of $34.3_{-0.4}^{+0.4} \mathrm{~W} / \mathrm{m} \cdot \mathrm{K}$ was obtained in the steady-state laser heating measurement. The relatively low measured thermal conductivity is attributed to numerous CNT-glue matrix and CNT-CNT thermal contact resistance. Compared with steady-state Raman method, the transient method does not require the detailed laser absorption value and additional temperature calibration experiment. Furthermore, the comprehensive Raman thermometry based on a single laser features 
simple configuration and fast thermal characterization for the self-heating and self-probing characteristics. The successful measurement of a composite CNT fiber proves that transient Raman method has powerful measurement ability and can be expanded for further applications. 


\section{Acknowledgements}

The authors thank the financial support from the National Natural Science Foundation of China

(Nos. 51428603 and 51576145), DOE (DENE) (0000671) and Iowa Energy Center (OG-15-011). 


\section{References}

[1] Balandin AA, Ghosh S, Bao W, Calizo I, Teweldebrhan D, Miao F, et al. Superior Thermal Conductivity of Single-Layer Graphene. Nano Lett 2008;8(3):902-7.

[2] Yue Y, Eres G, Wang X, Guo L. Characterization of thermal transport in micro/nanoscale wires by steady-state electro-Raman-thermal technique. Appl Phys A 2009;97(1):19-23.

[3] Doerk GS, Carraro C, Maboudian R. Single nanowire thermal conductivity measurements by Raman thermography. ACS Nano 2010;4(8):4908-14.

[4] Sahoo S, Gaur APS, Ahmadi M, Guinel JF, Katiyar RS. Temperature-Dependent Raman Studies and Thermal Conductivity of Few-Layer MoS2. J Phys Chem C 2013;117(17):9042-7.

[5] Yue Y, Wang X. Nanoscale thermal probing. Nano Rev 2012;3:11586.

[6] Li Q, Liu C, Wang X, Fan S. Measuring the thermal conductivity of individual carbon nanotubes by the Raman shift method. Nanotechnology 2009;20(14):5886-90.

[7] Hsu IK, Pettes MT, Bushmaker A, Aykol M, Li S, Cronin SB. Optical Absorption and Thermal Transport of Individual Suspended Carbon Nanotube Bundles. Nano Lett 2009;9(2):590-4.

[8] Malekpour H, Chang KH, Chen JC, Lu CY, Nika DL, Novoselov KS, et al. Thermal Conductivity of Graphene Laminate. Nano Lett 2014;14(9):5155-61.

[9] Lee JU, Yoon D, Kim H, Sang WL, Cheong H. Thermal conductivity of suspended pristine graphene measured by Raman spectroscopy. Phys Rev B 2011;83(8):3002-5.

[10] Chen S, Moore AL, Cai W, Suk JW, An J, Mishra C, et al. Raman Measurements of Thermal Transport in Suspended Monolayer Graphene of Variable Sizes in Vacuum and Gaseous 
Environments. ACS Nano 2011;5(1):321-8.

[11] Xu S, Tang X, Yue Y, Wang X. Sub-micron imaging of sub-surface nanocrystalline structure in silicon. J Raman Spectrosc 2013;44(11):1523-8.

[12] Yan Z, Jiang C, Pope TR, Tsang CF, Stickney JL, Goli P, et al. Phonon and thermal properties of exfoliated TaSe2 thin films. J Appl Phys 2013;114(20):204301.

[13] Zhang J, Hong Y, Yue Y. Thermal transport across graphene and single layer hexagonal boron nitride. J Appl Phys 2015;117(13):134307.

[14] Li Q-Y, Zhang X. T-type Raman spectroscopy method for determining laser absorption, thermal conductivity and air heat transfer coefficient of micro/nano fibers. Thermochim Acta 2014;581(6):26-31.

[15] Beechem T, Graham S, Kearney SP, Phinney LM, Serrano JR. Invited Article: Simultaneous mapping of temperature and stress in microdevices using micro-Raman spectroscopy. Rev Sci Instrum 2007;78(6):061301.

[16] Beechem T, Yates L, Graham S. Invited Review Article: Error and uncertainty in Raman thermal conductivity measurements. Rev Sci Instrum 2015;86(4):041101.

[17] Yue Y, Chen X, Wang X. Noncontact Sub-10 nm Temperature Measurement in Near-Field Laser Heating. ACS Nano 2011;5(6):4466-75.

[18] Parker WJ, Jenkins RJ, Butler CP, Abbott GL. Flash Method of Determining Thermal Diffusivity, Heat Capacity, and Thermal Conductivity. J Appl Phys 1961;32(9):1679-84.

[19] Tetsuya B, Ono A. Improvement of the laser flash method to reduce uncertainty in thermal diffusivity measurements. Meas Sci Technol 2001;12(12):2046-57. 
[20] Paddock CA, Eesley GL. Transient thermoreflectance from thin metal films. J Appl Phys 1986;60(1):285-90.

[21] Capinski W, Maris H, Ruf T, Cardona M, Ploog K, Katzer D. Thermal-conductivity measurements of GaAs/AlAs superlattices using a picosecond optical pump-and-probe technique. Phys Rev B 1999;59(12):8105.

[22] Schmidt AJ, Chen X, Chen G. Pulse accumulation, radial heat conduction, and anisotropic thermal conductivity in pump-probe transient thermoreflectance. Rev Sci Instrum 2008;79(11):114902.

[23] Yue Y, Zhang J, Wang X. Micro/nanoscale spatial resolution temperature probing for the interfacial thermal characterization of epitaxial graphene on 4H-SiC. Small 2011;7(23):3324-33.

[24] Cai W, Moore AL, Zhu Y, Li X, Chen S, Shi L, et al. Thermal Transport in Suspended and Supported Monolayer Graphene Grown by Chemical Vapor Deposition. Nano Lett 2010;10(5):1645-51.

[25] Tang X, Shen X, Zhang J, Wang X. Five Orders of Magnitude Reduction in Energy Coupling across Corrugated Graphene/Substrate Interfaces. ACS Appl Mater Inter 2014;6(4):2809-18.

[26] Tang X, Xu S, Wang X. Corrugated Epitaxial Graphene/SiC Interface: Photon Excitation and Probing. Nanoscale 2014;6(15):8822-30.

[27] Yue Y, Zhang J, Tang X, Xu S, Wang X. Thermal Transport across Atomic-Layer Material Interfaces. Nanotechnol Rev 2015;4:533-55.

[28] Liu J, Wang H, Hu Y, Ma W, Zhang X. Laser flash-Raman spectroscopy method for the 
measurement of the thermal properties of micro/nano wires. Rev Sci Instrum 2015;86(1):014901. [29] Xu S, Wang T, Hurley D, Yue Y, Wang X. Development of time-domain differential Raman for transient thermal probing of materials. Opt Express 2015;23:10040-56.

[30] Lu W, Zu M, Byun J-H, Kim B-S, Chou T-W. State of the Art of Carbon Nanotube Fibers: Opportunities and Challenges. Adv Mater 2012;24(14):1805-33.

[31] Zhang X, Li Q, Holesinger TG, Arendt PN, Huang J, Kirven PD, et al. Ultrastrong, Stiff, and Lightweight Carbon-Nanotube Fibers. Adv Mater 2007;19(23):4198-201.

[32] Koziol K, Vilatela J, Moisala A, Motta M, Cunniff P, Sennett M, et al. High-performance carbon nanotube fiber. Science 2007;318(5858):1892-5.

[33] Ericson LM, Fan H, Peng H, Davis VA, Zhou W, Sulpizio J, et al. Macroscopic, neat, single-walled carbon nanotube fibers. Science 2004;305(5689):1447-50.

[34] Li M, Yue Y. Raman-Based Steady-State Thermal Characterization of Multiwall Carbon Nanotube Bundle and Buckypaper. J Nanosci Nanotechno 2015;15:3004-10.

[35] Li Q, Li Y, Zhang X, Chikkannanavar SB, Zhao Y, Dangelewicz AM, et al. Structure-dependent electrical properties of carbon nanotube fibers. Adv Mater 2007;19(20):3358-63.

[36] Graf D, Molitor F, Ensslin K, Stampfer C, Jungen A, Hierold C, et al. Spatially resolved Raman spectroscopy of single-and few-layer graphene. Nano Lett 2007;7(2):238-42.

[37] DiLeo RA, Landi BJ, Raffaelle RP. Purity assessment of multiwalled carbon nanotubes by Raman spectroscopy. J Appl Phys 2007;101(6):064307.

[38] Zhang Y, Xie L, Zhang J, Wu Z, Liu Z. Temperature coefficients of Raman frequency of 
individual single-walled carbon nanotubes. J Phys Chem C 2007;111(38):14031-4.

[39] Guo J, Wang X, Wang T. Thermal Characterization Of Microscale Conductive And Nonconductive Wires Using Transient Electrothermal Technique. J Appl Phys 2007;101(6):063537.

[40] Li M, Li C, Wang J, Xiao X, Yue Y. Parallel measurement of conductive and convective thermal transport of micro/nanowires based on Raman mapping. Appl Phys Lett 2015;106(25):253108.

[41] Li M, Sun Y, Xiao H, Hu X, Yue Y. High temperature dependence of thermal transport in graphene foam. Nanotechnology 2015;26(10):105703.

[42] Yue Y, Liu K, Li M, Hu X. Thermal manipulation of carbon nanotube fiber by mechanical stretching. Carbon 2014;77(2):973-9.

[43] Xie H, Cai A, Wang X. Thermal diffusivity and conductivity of multiwalled carbon nanotube arrays. Phys Lett A 2007;369(1):120-3.

[44] Liu G, Lin H, Tang X, Bergler K, Wang X. Characterization of Thermal Transport in One-dimensional Solid Materials. J Vis Exp 2014(83):e51144. 